\title{
Protecting young children from influenza
}

\author{
Peter J. Gill ${ }^{1,2}$ and Kay Wang ${ }^{3}$
}

Affiliations: ${ }^{1}$ Division of Paediatric Medicine, Dept of Paediatrics, The Hospital for Sick Children, Toronto, ON, Canada. ${ }^{2}$ Dept of Paediatrics, University of Toronto, Toronto, ON, Canada. ${ }^{3}$ Nuffield Department of Primary Care Health Sciences, University of Oxford, Oxford, UK.

Correspondence: Peter J. Gill, The Hospital for Sick Children, 555 University Avenue, Toronto, ON, Canada, M5G 1X8. E-mail: peter.gillamail.utoronto.ca

@ERSpublications

Evidence-based strategies are needed to ensure children targeted by influenza programmes are vaccinated http://ow.ly/qap430evC7y

Cite this article as: Gill PJ, Wang K. Protecting young children from influenza. Eur Respir J 2017; 50: 1701550 [https://doi.org/10.1183/13993003.01550-2017].

Influenza is a major global cause of childhood morbidity and mortality $[1,2]$, and puts a strain on healthcare resources, particularly during epidemics and pandemics [3, 4]. Annual seasonal influenza vaccination programmes were launched to help reduce the burden of influenza but there is variation between countries on which children are targeted. Certain national programmes (for example, in Canada and the USA) recommend universal influenza vaccination $[5,6]$ while most European countries still focus mainly on groups considered to be at greater risk of influenza-related complications [7]. These include children with certain known underlying medical conditions and those under 2 years of age $[8,9]$.

In this issue of the European Respiratory Journal, HARDELID et al. [10] provide evidence to inform influenza vaccine strategies by identifying risk factors for influenza-related hospital admission in children younger than 2 years of age. The cohort study of 402762 singleton livebirths in Scotland from 2007 to 2015 used data linkages between birth and death registration, hospital administrative data and influenza laboratory reports from 2009 to 2015 (six influenza seasons). They identified 1019 influenza-confirmed hospital admissions during the study period, or 2.57 per 1000 child-years in children aged less than 6 months and 2.07 per 1000 child years in children aged 6-23 months [10]. The study findings highlight several limitations of current influenza immunisation programmes.

\section{Problems with current vaccination strategies \\ Targeting "at-risk" groups is likely to have limited impact on hospitalisations}

HARDELID et al. [10] found that $85 \%$ of influenza-related hospital admissions in children aged 6-23 months occurred in those who were not known to be in a clinical risk group. In addition, targeting preventative strategies at these risk groups would only prevent 3-6\% of hospital admissions. Nevertheless, targeting "at-risk" groups may be of value in prioritising resources during periods of high influenza activity, and in reducing burden of illness associated with other outcomes not examined by HARDELID et al. [10], including complications managed in the community and prolonged hospital admissions.

\section{Guidelines for vaccination of household contacts are inconsistent}

HARDELID et al. [10] found that having one or more siblings was the strongest predictor for hospital admission in infants aged less than 6 months. Infants aged under 6 months are at particularly high risk of influenza-related complications as no influenza vaccines are currently licensed for use in this age group [11], and vaccinating pregnant women has only a limited protective effect $[12,13]$. However, unlike in 
Canada and the USA [5, 6], only seven of 30 European Union member states recommend influenza vaccination for household contacts of infants aged under 6 months [7]. The findings reported by HARDELID et al. [10] therefore suggest that more widespread adoption of strategies involving the vaccination of older siblings of young infants should be considered.

\section{Delays incorporating new risk factors}

HARDELID et al. [10] also found that children aged 6-23 months who had been born prematurely had a $47 \%$ increased risk of influenza-related hospitalisations. This is consistent with the findings of a systematic review of published and unpublished data, which found that premature birth was a risk factor for hospitalisation in children presenting with influenza or influenza-like illness (ILI) in community-based clinical settings (mainly hospital ambulatory care) [8]. Yet children born prematurely are still not targeted by most influenza vaccine programmes $[6,7]$ even though an estimated 15 million children are born prematurely each year, ranging from $5 \%$ in several European countries to $12 \%$ in the USA [14].

\section{Implications for future research}

The study by HARDELID et al. [10] is one of the largest to date of influenza-confirmed hospital admissions in young children and the first to examine influenza risk factors in children less than 6 months of age [10]. It demonstrates the power of data linkage studies, with 596174 person child-years of follow-up spanning six influenza seasons. However, by only evaluating hospitalised children with confirmed influenza, the study did not address the potential impact of influenza vaccination on burden of illness in primary care $(e$. g. number of general practitioner visits) or the impact of illness on families (e.g. workplace leave for parents). Further data are therefore needed on risk factors relevant to primary care, and risk factors for influenza-related complications managed in the community.

It is likely that HaRDELID et al. [10] would have underestimated the number of influenza cases in their study population given that most hospitalised children with suspected respiratory infections are not tested for viruses [10]. Clear consensus on who and when to test for influenza (or other viruses) are lacking, including the role of point-of-care tests. One approach, as suggested in a recent review of paediatric respiratory virus testing, is to reserve influenza testing for children at increased risk of influenza-related complications [15]. Most clinicians use diagnostic tests to identify a subset of children who may benefit from the efficient use of antiviral medications. While the neuraminidase inhibitor oseltamivir has limited effectiveness in otherwise healthy children $[16,17]$, most guidelines recommend oseltamivir in "at-risk" children due to the increased risk of influenza-related complications and death in this population $[8,9,18]$. However, more recent data from 1725 children included in an individual participant data meta-analysis found no survival benefit in "at-risk" children [19]. High-quality randomised controlled trials are needed to define the populations that would benefit the most, if at all, from viral testing and antiviral therapy.

Controversy also still exists regarding the effectiveness of universal versus targeted vaccination programmes. Which one is more effective? Is effectiveness defined by a reduced number of cases, reduced complications or reduced cost? There is a clear tension between targeting "at-risk" children and reducing the overall burden of influenza on the healthcare system. Universal vaccination may draw attention away from greater resources spent focusing on a smaller number of "at-risk" patients, who have a greater risk of influenza-related complications. A policy of universal vaccination may only be worthwhile if influenza vaccination is effective at reducing complications and hospitalisations from influenza. Findings from a recent systematic review suggest that influenza vaccination of children may be effective at reducing rates of laboratory-confirmed influenza infection in some community settings, but more evidence is needed to determine which settings are most suitable for this strategy and whether targeting these settings would also result in reduction in complications and hospitalisations from influenza/ILI [20].

For both universal and targeted vaccination strategies, influenza vaccination uptake rates are much lower than those observed in relation to other childhood vaccinations, ranging 24-52.5\% [21, 22]. Reasons for poor uptake are multifactorial, relating to caregiver priority, access, perceived importance, understanding of indication and health beliefs, amongst others [23]. Evidence-based strategies to improve implementation of vaccination programmes are therefore needed to address these potential barriers, particularly in high priority and "difficult to reach" groups.

\section{References}

1 Wong KK, Jain S, Blanton L, et al. Influenza-associated pediatric deaths in the United States, 2004-2012. Pediatrics 2013; 132: 796-804.

2 Cromer D, van Hoek AJ, Jit M, et al. The burden of influenza in England by age and clinical risk group: a statistical analysis to inform vaccine policy. J Infect 2014; 68: 363-371.

3 Neuzil KM, Mellen BG, Wright PF, et al. The effect of influenza on hospitalizations, outpatient visits, and courses of antibiotics in children. $N$ Engl J Med 2000; 342: 225-231. 
4 Van Kerkhove MD, Vandemaele KA, Shinde V, et al. Risk factors for severe outcomes following 2009 influenza A (H1N1) infection: a global pooled analysis. PLoS Med 2011; 8: e1001053.

5 Grohskopf LA, Sokolow LZ, Broder KR, et al. Prevention and control of seasonal influenza with vaccines. MMWR Recomm Rep 2016; 65: 1-54.

6 National Advisory Committee on Immunization. Canadian immunization guide chapter on influenza and statement on seasonal influenza vaccine for 2016-2017. http://www.phac-aspc.gc.ca/naci-ccni/flu-2016-grippe-eng. php Date last updated: October 18 2016. Date last accessed: July 22017.

7 European Centre for Disease Prevention and Control. Seasonal influenza vaccination and antiviral use in Europe: overview of vaccination recommendations and coverage rates in the EU Member States for the 2013-14 and 201415 influenza seasons. https:/ecdc.europa.eu/sites/portal/files/media/en/publications/Publications/Seasonalinfluenza-vaccination-antiviral-use-europe.pdf Date last updated: July 2016. Date last accessed: July 92017.

8 Gill PJ, Ashdown HF, Wang K, et al. Identification of children at risk of influenza-related complications in primary and ambulatory care: a systematic review and meta-analysis. Lancet Respir Med 2015; 3: 139-149.

9 Dalziel SR, Thompson JM, Macias CG, et al. Predictors of severe H1N1 infection in children presenting within Pediatric Emergency Research Networks (PERN): retrospective case-control study. BMJ 2013; 347 : f4836.

10 Hardelid P, Verfuerden M, McMenamin J, et al. Risk factors for admission to hospital with laboratory-confirmed influenza in young children: birth cohort study. Eur Respir J 2017; 50: 1700489.

11 Bonati M, Clavenna A. Seasonal influenza immunization in early infancy? BMC Public Health 2012; 12 : 873.

12 Shakib JH, Korgenski K, Presson AP, et al. Influenza in infants born to women vaccinated during pregnancy. Pediatrics 2016; 137: e20152360.

13 Nunes MC, Cutland CL, Jones S, et al. Duration of infant protection against influenza illness conferred by maternal immunization: secondary analysis of a randomized clinical trial. JAMA Pediatr 2016; 170: 840-847.

14 Blencowe $\mathrm{H}$, Cousens S, Oestergaard MZ, et al. National, regional, and worldwide estimates of preterm birth rates in the year 2010 with time trends since 1990 for selected countries: a systematic analysis and implications. Lancet 2012; 379: 2162-2172.

15 Gill PJ, Richardson SE, Ostrow O, et al. Testing for respiratory viruses in children: to swab or not to swab. JAMA Pediatr 2017; 171: 798-804.

16 Wang K, Shun-Shin M, Gill P, et al. Neuraminidase inhibitors for preventing and treating influenza in children (published trials only). Cochrane Database Syst Rev 2012; 4: CD002744.

17 Jefferson T, Jones MA, Doshi P, et al. Neuraminidase inhibitors for preventing and treating influenza in healthy adults and children. Cochrane Database Syst Rev 2014; 4: CD008965.

18 Sachedina N, Donaldson LJ. Paediatric mortality related to pandemic influenza A H1N1 infection in England: an observational population-based study. Lancet 2010; 376: 1846-1852.

19 Muthuri SG, Venkatesan S, Myles PR, et al. Effectiveness of neuraminidase inhibitors in reducing mortality in patients admitted to hospital with influenza A H1N1pdm09 virus infection: a meta-analysis of individual participant data. Lancet Respir Med 2014; 2: 395-404.

20 Yin JK, Heywood AE, Georgousakis M, et al. Systematic review and meta-analysis of indirect protection afforded by vaccinating children against seasonal influenza: implications for policy. Clin Infect Dis 2017; 65: 719-728.

21 Rodgers L, Pabst LJ, Chaves SS. Increasing uptake of live attenuated influenza vaccine among children in the United States, 2008-2014. Vaccine 2015; 33: 22-24.

22 Rajaram S, Steffey A, Blak B, et al. Uptake of childhood influenza vaccine from 2012-2013 to 2014-2015 in the UK and the implications for high-risk children: a retrospective observational cohort study. BMJ Open 2016; 6: e010625.

23 Sampson R, Wong L, Macvicar R. Parental reasons for non-uptake of influenza vaccination in young at-risk groups: a qualitative study. Br J Gen Pract 2011; 61: e386-e391. 